\title{
Résolution de problèmes mathématiques ouverts dans un espace virtuel 3D : cas de l'environnement ANIPPO
}

\section{Solving open mathematical problems in a 3D virtual space: Case of the ANIPPO environment}

\author{
Richard Cabassut ${ }^{1 *}$, Claude-Alexandre Magot $^{1}$, Ecaterina Pacurar $^{2}$, and Yohan \\ Solon ${ }^{2}$ \\ ${ }^{1}$ LISEC-EA2310, INSPE Université de Strasbourg, France. \\ ${ }^{2}$ CIREL-Trigone, Université de Lille, France
}

\begin{abstract}
Résumé. Des recherches ont montré l'intérêt et les limites de la manipulation dans l'enseignement des mathématiques [1]. Dans le contexte de l'apparition d'environnements virtuels et de la place centrale de la résolution de problèmes, nous étudions la manipulation virtuelle, dans la résolution de problèmes ouverts de mathématiques, dans l'environnement ANIPPO, par des élèves de la fin de l'école primaire. La méthodologie permet de comparer un groupe expérimental dans l'environnement ANIPPO avec un groupe de contrôle dans l'environnement traditionnel. Nous décrivons le cadre théorique des problèmes ouverts, des registres de représentations sémiotiques, de la collaboration et de la motivation. Nous précisons comment les résultats de la pré-expérimentation montrent les difficultés des apprentissages dans un environnement virtuel a-didactique et influencent les conditions de l'expérimentation à venir, pour l'appropriation de l'environnement ANIPPO, la prise en compte des procédures des élèves et la progressivité des problèmes à résoudre.
\end{abstract}

\begin{abstract}
Research has shown the interest and the limits of manipulation in the teaching of mathematics [1]. In the context of the emergence of virtual environments and the central place of problem solving, we study virtual manipulation, in solving open mathematical problems, in the ANIPPO environment, by students at the end of primary school. The methodology makes it possible to compare an experimental group in the ANIPPO environment with a control group in the traditional environment. We describe the theoretical framework of open problems, registers of semiotic representations, collaboration and motivation. We specify how the results of the pre-experiment show the difficulties of learning in a virtual a-didactic environment and influence the conditions of the future experiment, for the
\end{abstract}

\footnotetext{
* Corresponding author: richard.cabassut@unistra.fr
} 
appropriation of the ANIPPO environment, the development of pupils' procedures and the progressiveness of problems to be solved.

\section{Introduction : Quels apports de l'environnement virtuel ANIPPO dans la résolution de problèmes ouverts ?}

Dans cette introduction nous précisons le contexte et les objectifs de cette recherche. La manipulation dans l'apprentissage des mathématiques, que ce soit dans un environnement réel ou virtuel, est un élément important dans l'apprentissage des mathématiques $[2,3]$. La résolution de problèmes continue d'occuper une place centrale dans le curriculum français $[4,5]$. Les nouveaux programmes de la scolarité obligatoire mis en œuvre en France en septembre 2016 soulignent l'importance de la résolution de problèmes pour donner du sens aux apprentissages, et les compétences "modéliser" et "chercher", signalées parmi les six compétences mathématiques essentielles, valorisent la pratique du problème ouvert. De plus l'importance du travail en environnement numérique est encouragée dès l'école primaire, avec le développement de l'algorithmique et de la programmation, et le développement des environnements numériques (plateformes, logiciels, robots ...). La recherche en didactique des mathématiques en France montre que le thème de la résolution de problèmes reste d'actualité $[6,7]$. Le thème de la géométrie en lien avec les représentations et les artefacts vient d'être récemment traité dans les colloques de la COPIRELEM d'Epinal (2017) et de Blois (2018) sur la formation et l'enseignement des mathématiques à l'école primaire. Depuis les travaux de l'institut national de la recherche pédagogique (INRP), en 1972, avec l'équipe ERMEL, la résolution des problèmes est au cœur des apprentissages de l'école primaire et soutient la pratique de la collaboration entre élèves: " the wish is also to develop a cooperative activity among the students and to limit the interventions of the teacher $\gg$ [8]. Une équipe pluridisciplinaire rassemblant des spécialistes des sciences de l'éducation, des sciences cognitives, de la didactique des mathématiques et de la didactique de l'informatique ont élaboré un projet de recherche. Ce projet est retenu et financé par l'Agence Nationale de Recherche (ANR) française. Les deux objectifs principaux de ce projet de recherche sont d'une part d'analyser l'articulation entre compréhension du monde virtuel manipulé et la compréhension des savoirs présentés par celui-ci, et d'autre part d' étudier l'impact de l'approche coopérative, de l'entraide et de la pédagogie de l'erreur sur le processus d'apprentissage de résolution de problèmes « ouverts » dont les contenus sont formalisés et intégrés dans un environnement virtuel 3D, ANIPPO (Application Numérique Interactive Pour des Problèmes Ouverts).

\section{Cadre théorique : Représentations, collaboration et motivation lors de la résolution de problèmes ouverts dans un environnement virtuel}

Avec les registres de représentations sémiotiques de [9], les résultats de la recherche montrent l'importance des représentations dans l'enseignement et l'apprentissage des mathématiques [10]. L'environnement ANIPPO permettra de travailler dans différents registres de représentation [9] :

- le registre du monde virtuel ANIPPO où différents traitements sont possibles: le déplacement d'un avatar dans l'environnement par des commandes au clavier, le contrôle visuel à l'écran d'un déplacement virtuel d'un avatar, l'exploration visuelle à l'écran d'un 
lieu virtuel (par exemple en déplaçant l'avatar autour d'un solide virtuel ou à l'intérieur d'un solide virtuel),

- le registre de la langue naturelle écrite dans les fenêtres prévues pour répondre aux problèmes posés, ou dans les causeries (chats) entre élèves, dans lequel par exemple des élèves peuvent échanger des informations en faisant référence à des repères de l'espace virtuel,

- le registre de la langue orale lors des échanges entre élèves.

Duval nous invite à distinguer les traitements intra-registres (comme le déplacement à l'intérieur de l'environnement ANIPPO, ou encore le dénombrement de petits cubes par un calcul sur des nombres écrits en chiffres) et les traitements de conversion inter-registres (comme la description en langue naturelle d'un déplacement dans l'environnement ANIPPO ou le repérage en langue naturelle d'un objet situé dans l'espace virtuel ANIPPO): «Mathematical comprehension begins when coordination of registers starts up. [...] Mathematical thinking processes depend on a cognitive synergy of registers of representation» [9].

Dans notre recherche, les élèves recourent à ces représentations et à ces échanges dans le cas d'activités de résolution de problèmes qui occupent une place centrale dans le curriculum français [8]. Ces activités concernent des problèmes ouverts : "Open problems are there defined as problems with a short text, which induces neither a method nor a solution; their solving does not reduce to the direct application of known results or tools; they situate in a context familiar enough to the students to make the problem meaningful, and allow them to engage in trials, conjectures »[8].

Concernant la collaboration et la coopération nous utiliserons le cadre théorique des travaux de Baudrit $[11,12]$. Nous considérons que la situation de résolution de problème est collaborative « à partir du moment où des personnes de même niveau cognitif, dont les statuts sont équivalents, sont capables de travailler ensemble dans un but commun. Cette situation est ensuite considérée comme interactive si ces personnes communiquent de façon soutenue, argumentent, voire s'opposent en évitant toutefois d'imposer leurs points de vue » [12]. Or dans l'environnement ANIPPO les élèves auront la possibilité de se reconnaître dans l'espace virtuel où leurs avatars circulent et peuvent échanger entre eux à l'aide d'un casque audio associé à un micro, ou par écrit à l'aide d'une causerie (chat). Nous observerons si des collaborations et des entraides se mettent en place dans cet environnement a-didactique (absence du professeur).

Pour Lieury et Fenouillet [13] « la motivation désigne une force intra-individuelle qui peut avoir des déterminants internes et/ou externes et qui permet d'expliquer la direction, le déclenchement, la persistance et l'intensité du comportement ou de l'action [...]. Cette force provoque quatre effets : - le déclenchement d'un comportement; - l'orientation du comportement, attirance vers un but ou au contraire rejet ou fuite; - l'intensité de la mobilisation énergétique, émotion, attention ; - et enfin la persistance du comportement dans le temps ». Nous observerons si l'environnement ANIPPO favorise la motivation à la résolution de problèmes.

Avec ce cadre théorique nous formulons donc les trois hypothèses de recherche suivantes à vérifier.

$\boldsymbol{H 1}$ : L'immersion de l'élève dans le monde virtuel renforce le caractère a-didactique par identification avec son avatar et par une meilleure relation du sujet au monde réel.

$\boldsymbol{H} 2$ : Le renforcement de la situation a-didactique facilite la transition vers des stratégies procédurales élaborées à partir des savoirs mathématiques impliqués.

$\boldsymbol{H 3}$ : La motivation à la résolution de problèmes ouverts va augmenter dans le cadre d'une mise en situation en environnement immersif 3D. 
Décrivons maintenant la méthodologie suivie pour vérifier les hypothèses.

\section{Méthodologie : de la pré-expérimentation à l'expérimentation}

La méthodologie consiste à mettre en œuvre une pré-expérimentation (en 2019) dont les résultats permettront de préciser une expérimentation (en 2020). Dans les deux cas on compare les résolutions de problèmes entre des élèves d'un groupe de contrôle et des élèves d'un groupe expérimental. Les élèves des deux classes doivent résoudre les mêmes problèmes mais dans des conditions différentes.

Dans la groupe de contrôle les élèves sont en configuration normale de classe, en présence de leur professeur, avec du matériel concret (des petits cubes qui s'emboîtent) et différentes photos d'un solide, de différents points de vue (de gauche, de face, et de dessus).

Dans le groupe expérimental chaque élève travaille (dans une salle informatique) seul devant un ordinateur, dans lequel est implanté l'environnement d'espace virtuel 3D ANIPPO. Il n'y a pas le professeur de classe (situation a-didactique). Les élèves peuvent communiquer entre eux avec un casque audio muni d'un micro, ou par écrit dans une causerie (chat) disponible dans l'environnement ANIPPO.

Chacune des classes (de contrôle ou expérimentale) a trois sessions de travail.

\subsection{Première session}

Une première session d'évaluation initiale poursuit deux objectifs. D'une part il s'agit de s'assurer que les élèves ont les connaissances minimales pré-requises pour pouvoir résoudre les problèmes proposés. Il s'agit essentiellement de connaissances portant sur les noms de solides usuels et sur les notions liées : sommet, arête et face. D'autre part un problème à résoudre est proposé : il s'agit de mesurer la performance de chaque élève dans la résolution du problème, pour éventuellement observer s'il y a un lien entre les performances de l'élève dans l'évaluation et son habileté à réaliser les tâches proposées dans les deux sessions suivantes. D'autre part la correction de cette évaluation initiale doit être effectuée avant les autres sessions pour s'assurer que les élève ont bien eu un enseignement minimal des prérequis. Voici les consignes et questions de l'évaluation.

Question 1. Le professeur distribue à chaque élève un pavé droit (par exemple un élément d'un jeu de construction en bois ou 3 petits cubes emboités). Chaque élève doit se munir d'un stylo qui ne s'efface pas. L'idée est que chaque élève ait le même temps pour répondre à une question et ne soit pas perturbé par la question suivante. Consigne : Vous avez 5 minutes pour toucher le solide que je vous ai distribué puis pour répondre. Une fois que vous avez répondu vous retourner votre feuille et vous posez votre stylo sur la table. Puis chaque élève reçoit une feuille de réponses à remplir et dispose alors de 5 minutes puis les feuilles sont ramassées.

Quel est le nom de ce solide?

Combien de sommets a-t-il ?

Combien d'arêtes a-t-il ?

Combien de faces a-t-il ?

Question 2. Consigne :

En regardant le dessin que j'affiche au tableau vous avez 5 minutes pour répondre. Une fois que vous avez répondu vous retourner votre feuille et vous posez votre stylo sur la table. Chaque élève reçoit une feuille de réponses à remplir et dispose alors de 5 minutes puis les feuilles sont ramassées. (L'enseignant affiche au tableau le dessin au tableau à une échelle convenable visible de tous) 


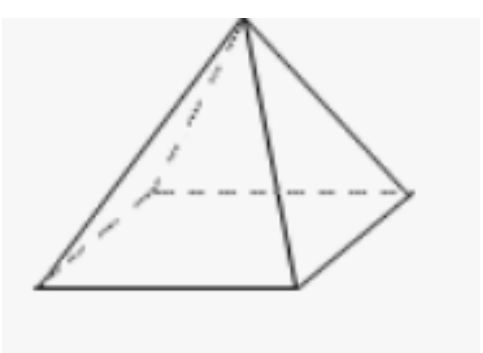

Fig. 1. Figure du solide.

Quel est le nom de ce solide ?

Combien de sommets a-t-il?

Combien d'arêtes a-t-il ?

Combien de faces a-t-il ?

Question 3. Consigne : Le professeur affiche l'énoncé suivant au tableau et le lit.

Calculer la longueur de ficelle nécessaire pour entourer la boite dessinée sur votre feuille, sachant qu'il faut $10 \mathrm{~cm}$ pour le nœud. Vous avez 15 minutes pour répondre. Chaque élève reçoit une feuille de réponses à remplir et dispose alors de 15 minutes puis les feuilles sont ramassées.

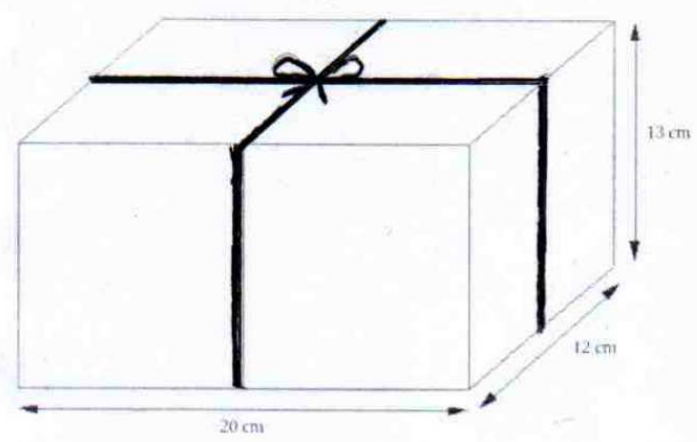

Fig. 2. Figure du problème.

Remarque : Il est conseillé de s'assurer de la compréhension de la consigne avant de distribuer les feuilles de réponse. Il doit bien dire aux élèves qu'il s'agit de savoir ce dont ils ont besoin et non pas de les noter.

\subsection{Seconde session}

Une seconde session d'appropriation vise à découvrir et s'approprier l'environnement de travail.

Dans le groupe de contrôle il s'agit d'une séance d'appropriation du matériel constitué par les petits cubes qui s'emboîtent. Des photos illustrent comment emboîter les cubes ou quelles constructions sont à réaliser pour s'approprier le matériel. 


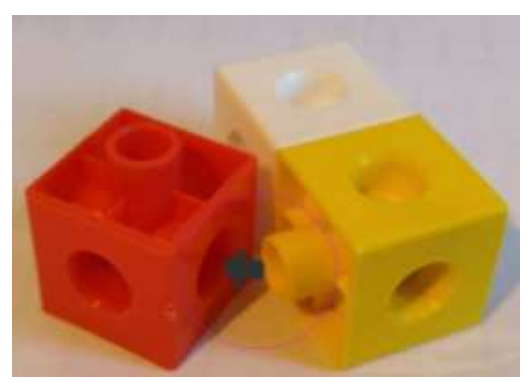

Fig. 3. Emboitements de cubes.

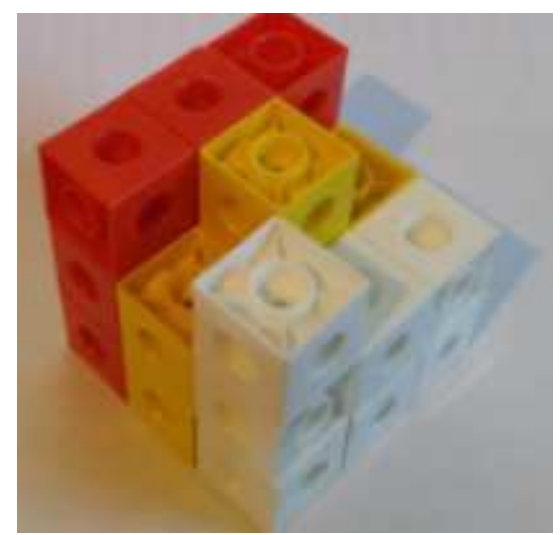

Fig. 4. Construction à reproduire.

Dans le groupe expérimental il s'agit d'une séance d'appropriation de l'environnement ANIPPO. Dans cette séance les élèves n'ont pas à résoudre de problèmes. Différentes captures d'écran illustrent comment se déplacer dans l'environnement, comment changer son avatar, comment utiliser le chat. Puis quelques parcours d'exploration de l'environnement sont proposés.

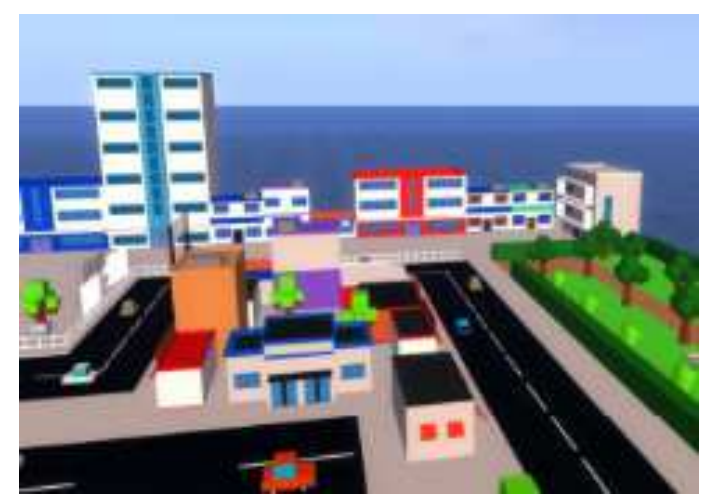

Fig. 5. Espace à explorer

\subsection{Troisième session}

Une troisième session est constituée par une séance de résolution de problèmes. 
Le groupe de contrôle travaille en configuration de classe normale, avec le matériel des petits cubes qui s'emboîtent. Chaque élève répond sur une feuille aux deux problèmes suivants. On considère un solide formé par un grand cube auquel on a enlevé des petits cubes. Voici quelques vues de ce solide.

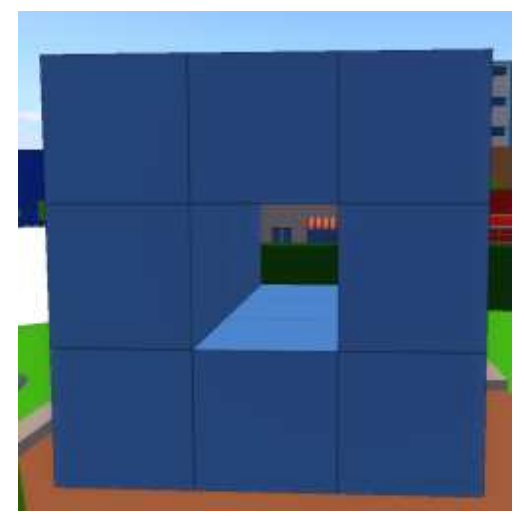

Fig. 6. Vue de face.

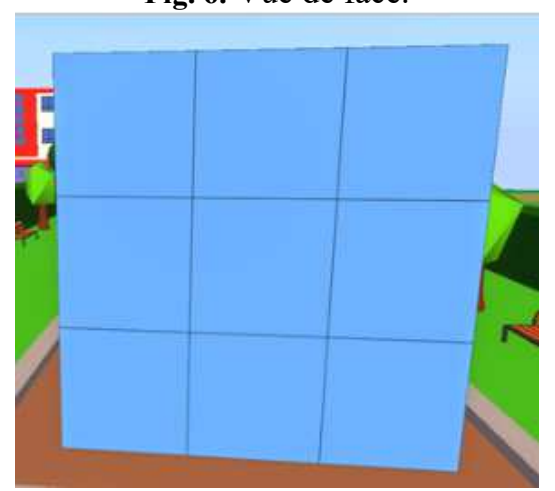

Fig. 7. Vue de gauche.

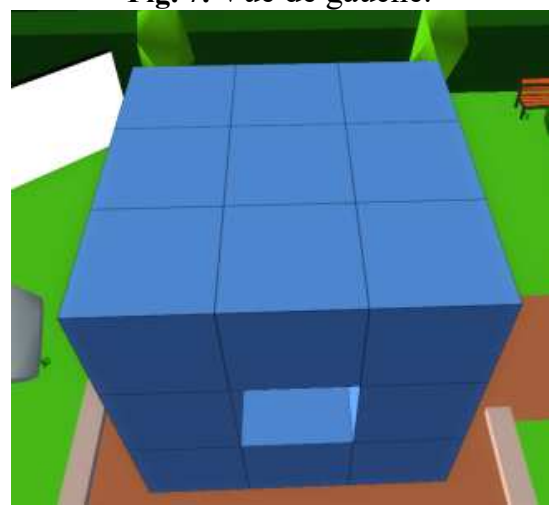

Fig. 8. Vue de dessus.

A) Combien de petits cubes bleus faut-il pour former le solide (formé par un grand cube auquel on a enlevé des petits cubes)?

B) Chaque petit cube a des faces carrées. Combien de faces carrées faut-il pour couvrir le solide (y compris dans la partie intérieure où on a enlevé des petits cubes) ?

Consignes: Si vous voulez, pour vous aider, vous pouvez utiliser des petits cubes emboîtables qui vous sont fournis lors de cette séance en classe. 
Le groupe expérimental travaille en salle d'informatique, chaque élève disposant d'un ordinateur sur lequel l'environnement ANIPPO est implanté. L'avatar peut donc se déplacer dans l'espace virtuel, autour du grand cube ainsi qu'à l'intérieur du grand cube.

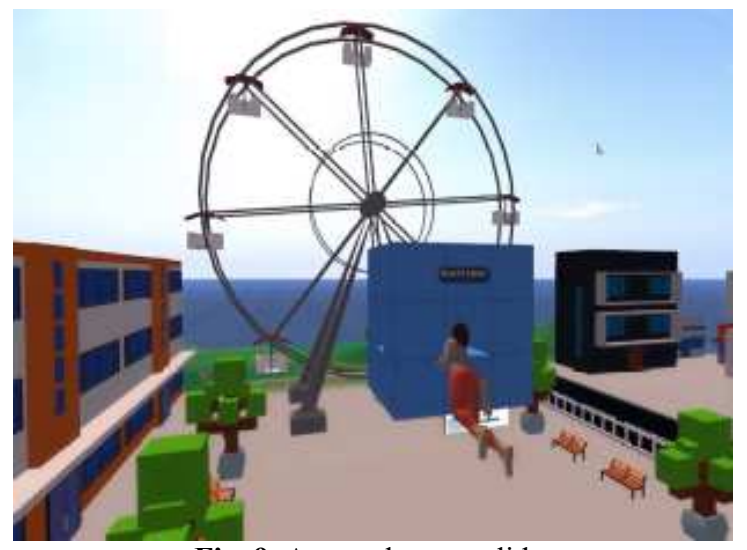

Fig. 9. Avatar devant solide.

A partir des résultats de la pré-expérimentation des ajustements seront effectués sur les différentes sessions d'évaluation, d'appropriation et de résolution de problèmes. Pour des questions techniques le test en psychologie sur la motivation n'a pas pu être mis en œuvre lors de la pré-expérimentation.

\section{Résultats de la pré-expérimentation et impact sur l'expérimentation}

Dans le groupe de contrôle composé de 26 élèves, la première question sur le nombre de petits cubes constituant le grand cube évidé 20 réponses sont correctes. D'après le détail des calculs on devine deux procédures. Le calcul « $8 \mathrm{X} 3=24$ » suggère que les élèves ont découpé le grand cube évidé en trois tranches verticales, chaque tranche étant constituée par un carré de 3 cubes sur 3, auquel on a enlevé le cube central, soit 9-1=8 par tranche, et 3x8 en tout. Un autre calcul «27-3=24 » suggère que les élèves ont compté le nombre de petits cubes d'un grand cube $3 \times 3 \times 3$ soit 27 cubes desquels ils ont retiré les 3 cubes de la partie centrale, soit $27-3=24$.

La seconde question dont la réponse correcte est 64 est réussie par seulement 4 élèves. La question ne semble pas avoir été comprise. Par exemple une réponse incorrecte est 27x6=162, car les 27 cubes précédents sont couverts chacun par 6 faces.

Dans le groupe expérimental, pour les deux questions, aucune réponse correcte n'est donnée.

\section{Discussion des résultats de la pré-expérimentation.}

Essayons d'interpréter ces résultats. Nous sommes d'abord très surpris de la différence de taux de réussite entre le groupe de contrôle et le groupe expérimental.

Nous pensons qu'ils traduisent d'une part une difficulté d'appropriation de l'environnement ANIPPO dans le temps imparti d'une session de 1h30. Les enregistrements des déplacements à l'écran des avatars montrent que beaucoup d'élèves ont de la difficulté à se déplacer dans l'environnement et ont de la difficulté à accomplir certaines actions. De même les élèves semblent ne pas prendre le temps de lire les consignes affichées dans l'environnement. Faudrait-il plutôt un message audio qui serait délivré au passage devant un détecteur? 
D'autre part la situation dans l'environnement ANIPPO apparaît très a-didactique, à tel point que beaucoup d'élèves semblent oublier les objectifs de résolution de problèmes initiaux : certains élèves s'amusent à explorer l'environnement et à jouer à cachecache entre avatars.

Concernant l'hypothèse $\mathrm{H} 1$, l'immersion de l'élève dans le monde virtuel semble renforcer de manière très forte le caractère a-didactique de la situation, par identification avec son avatar et par une meilleure relation du sujet au monde réel. Cette immersion apparaît même comme un obstacle en comparaison au groupe de contrôle. Il faudrait donc une modération du caractère a-didactique : peut-être que l'identification à l'avatar doit être plus distante pour éviter de trop distraire l'élève. Faut-il effectuer des rappels réguliers des objectifs de résolution du problème? Faut-il prévoir un temps d'appropriation plus long pour que la session de résolution de problèmes ne soit plus perturbée par une appropriation incomplète de l'environnement? Enfin, pour ce qui concerne le groupe de contrôle, est-ce qu'un phénomène de copiage entre élèves existe et qui serait favorisé par l'environnement de classe entière et par des dispositifs du type feuille de réponse à remplir, qui ne sont pas sans rappeler les dispositifs d'évaluation? Peut-être serait-il intéressant de noter la disposition géographique des élèves pour observer si un phénomène de réponses semblables (correcte $\mathrm{s}$ ou non) existe pour des élèves voisins?

Concernant l'hypothèse $\mathrm{H} 2$, pour les quelques élèves qui ont donné des réponses incorrectes à la première question, il a été difficile d'observer leurs procédures. Lors des déplacements dans l'environnement virtuel des informations sont échangées dans le registre de l'écrit, notamment l'affichage de l'énoncé du problème et la production par l'élève d'une solution au problème. Est-ce que ce passage du registre de l'action au registre de l'écrit est problématique dans un environnement virtuel ? Est-ce que l'attention et la motivation sont suffisants pour mémoriser l'énoncé écrit au cours des déplacements et pour mettre en place des explorations de l'environnement virtuel avec pour objectif la résolution du problème ? Il serait peut-être intéressant, une fois une réponse proposée par l'élève, d'enregistrer les justifications orales de son résultat, soit par un procédé d'enregistrement partie prenante de l'environnement ANIPPO, soit par un interrogatoire extérieur. Un dispositif de meilleures traces des justifications des réponses pourrait également être proposé pour le groupe de contrôle. L'environnement virtuel ANIPPO apparaît complexe et peu intuitif dans son design et son utilisation: une simplification permettrait-elle une appropriation plus rapide et meilleure?

Concernant l'hypothèse $\mathrm{H} 3$, la motivation à la résolution de problèmes ouverts semble au contraire avoir diminué avec l'environnement ANIPPO, qui semble apparaître comme un distracteur par rapport à la résolution de problèmes. Les échanges ou collaborations entre élèves, enregistrées dans le dispositif ANIPPO, portent davantage sur l'appropriation de l'environnement («comment fais-tu pour ... ? ») que sur la résolution de problèmes. Les difficultés techniques qui ont empêché de mettre en place les tests psychologiques sur la motivation, ne permettent pas de confirmer cette impression.

De nombreux problèmes techniques sont apparus dans les deux endroits (Lille et Marseille) où la pré-expérimentation s'est produite: il faudra résoudre ces problèmes avant l'expérimentation. Mais c'est une caractéristique des enseignements impliquant les TIC que la technique domine l'action didactique [14].

Concernant le second problème, très peu réussi dans le groupe de contrôle et pas réussi dans le groupe expérimental, la question du niveau de difficulté des problèmes de géométrie dans l'espace est posée. Pourtant les travaux de l'équipe ERMEL [15] semblent montrer que ce type de problèmes est accessible dès l'école primaire. S'agit-il donc d'un problème de compréhension de l'énoncé ? Les élèves du groupe de contrôle et du groupe expérimental 
ont-ils suffisamment travaillé ce domaine ? Il faudrait prévoir éventuellement des problèmes où la difficulté apparaît plus progressivement. L'évaluation prévue en première session n'a pas pu avoir lieu et n'a donc pas livré d'éclairage complémentaire. Il faudrait peut-être prévoir une session supplémentaire de résolution de problème pour proposer une montée progressive dans le niveau de difficulté des problèmes.

\section{Conclusion et perspectives}

Nous avons décrit jusqu'au stade de la pré-expérimentation un dispositif de recherche sur la résolution de problèmes ouverts à l'école primaire dans l'environnement virtuel 3D ANIPPO. Nous avons montré l'importance de plusieurs facteurs : le contexte technique qui devrait garantir un bon déroulement des expérimentations, le temps d'appropriation des environnements (concrets ou virtuels) qui doit être suffisant notamment pour l'environnement ANIPPO riche et complexe, les registres de représentation utilisés pour les échanges d'information, l'observation des justifications de stratégies et de procédures des élèves. Notamment pour la compréhension des énoncés de problèmes et pour l'observation des stratégies mises en place pour les résoudre et des procédures conduisant aux solutions, des dispositifs complémentaires doivent être proposés du type interrogatoire d'explicitation oral partie prenante de l'environnement ANIPPO ou extérieur à l'environnement. Dans les conditions de la pré-expérimentation, l'environnement ANIPPO est apparu peu favorable à la résolution de problèmes ouverts dans un espace 3D. Une modification des conditions d'expérimentation et des scénarios d'utilisation de cet environnement permettrait-elle de favoriser la résolution de problèmes ouverts et de voir apparaître des stratégies et des procédures de résolution spécifiques à cet environnement?

\section{Références}

1. Carbonneau, K. J., Marley, S. C., \& Selig, J. P., A meta-analysis of the efficacy of teaching mathematics with concrete manipulatives, Journal of Educational Psychology, 105, 380-400 (2013).

2. Moyer, P. S., Bolyard, J. J., \& Spikell, M. A., What are virtual manipulatives?, Teaching Children Mathematics, 8(6), 372-377 (2002).

3. Žilková, K., Edita Partová, E., Virtual manipulatives with cubes for supporting the learning process, in J. Novotna, \& H. Moraova (Eds.), International Symposium Elementary Maths Teaching SEMT'19 Proceedings, 427-437., Prague: Charles University, Faculty of Education (2019).

4. Villani, C., Torossian, C., 21 mesures pour l'enseignement des mathématiques, Ministère de l'Education Nationale (2018).

5. MEN (Ministère de l'Education Nationale). La résolution de problèmes à l'école élémentaire, Bulletin Officiel Spécial de l’Education Nationale $\mathrm{n}^{\circ} \mathbf{3}$ du 26 avril, 10-19 (2018).

6. Sander, E. , Une approche interprétative de la résolution de problèmes, in Julia Pilet \& Céline Vendeira (ed.) Préactes du séminaire de didactique des mathématiques. ARDM (2018).

7. Houdement, C., Problèmes arithmétiques basiques : le cour du problème? in Julia Pilet $\&$ Céline Vendeira (ed.) Préactes du séminaire de didactique des mathématiques. ARDM (2018).

8. Artigue M., Houdement C., Problem solving in France: didactic and curricular perspectives, ZDM Mathematics Education 39, 365-382, (2007). 
9. Duval, R., A cognitive analysis of problems of comprehension in a learning of mathematics. Educational Studies in Mathematics 61, 103-130 (2006).

10. Goldin, G. A., Representational systems, learning, and problem solving in mathematics, The Journal of Mathematical Behavior, 17(2), 137-165 (1998).

11. Baudrit, A., Apprentissage coopératif et entraide à l'école. Revue française de pédagogie, Vol. octobre - décembre, $\mathrm{n}^{\circ} 153$ (2005).

12. Baudrit, A., Apprentissage coopératif/Apprentissage collaboratif: d'un comparatisme conventionnel à un comparatisme critique, Revue Les sciences de l'Education- Pour l'èree nouvelle, 2007/1 Vol.40, p. 115 à 136 (2007).

13. Lieury, A., Fenouillet, F., Motivation et réussite scolaire, Dunod, Paris (2013).

14. Trestini, M., Cabassut, R., La domination de la technique sur l'action didactique et sur les scénarios pédagogiques en formation à distance, Colloque international TICE 2006. INP de Toulouse (2006).

15. Douaire, J. Emprin, F. Rajain, C., L'apprentissage du 3D à l'école, des situations d'apprentissage à la formation des enseignants, Repère $n^{\circ} 76$, Topic éditions, (2009). 